\title{
A New Test of Throughput Invariance in Fitts' Law: Role of the Intercept and of Jensen's Inequality
}

\author{
Halla B. Olafsdottir \\ Institut Mines-Telecom \\ Telecom ParisTech \\ CNRS LTCI \\ halla@telecom-paristech.fr
}

\author{
Yves Guiard \\ CNRS LTCI \\ Institut Mines-Telecom \\ Telecom ParisTech \\ yves.guiard@telecom-paristech.fr
}

\author{
Olivier Rioul \\ Institut Mines-Telecom \\ Telecom ParisTech \\ CNRS LTCI
}

\author{
Simon T. Perrault \\ Institut Mines-Telecom \\ Telecom ParisTech \\ CNRS LTCI
}

\begin{abstract}
Fitts' law states that movement time varies linearly with the index of difficulty or, equivalently, that throughput $(T P)$ is conserved across variations of the speed/accuracy strategy. Replicating a recent study by MacKenzie and Isokoski (2008), we tested the throughput invariance hypothesis with some fresh data and found the TP to be systematically affected by the strategy. This result, we suggest, pleads against the currently popular definition of the TP inherited from Fitts (1954), namely $T P=I D / M T$, which we recall is incompatible with the Shannon equation of Fitts' law. We also show that the statistical elaboration of the TP suffers from a problematic amount of uncontrolled variability due to the multiple inadvertent impact of Jensen's inequality.
\end{abstract}

Keywords: Throughput, Fitts' law, y-intercept, Jensen's inequality, statistics, aggregation order

\section{INTRODUCTION}

Humans have innumerable opportunities in everyday life to move their hand to some target location, for example to reach a light switch on a wall or to grasp some nearby object. In the specific context of human-computer interaction $(\mathrm{HCl})$, the ubiquitous graphical user interface requires the people to express almost all their decisions by reaching and clicking target objects like icons, menu items or hypertext links. In all these cases users face a speed/accuracy dilemma-as everyone knows, the faster the reaching movement, the more likely the miss. This speed/accuracy trade-off is what Fitts' law is all about.

In the present paper, concerned with both the mathematical consistency and the empirical validity of Fitts' law modeling, we focus on a seldomconsidered version of the law that takes the form of an invariance: if the equation is correct, a certain quantity, called the throughput, should be conserved across variations of the speed/accuracy balance. We will discuss, in light of some data, two difficulties that have hindered progress in the understanding of this conservation so far. One has to do with the controversial role of the equation's intercept and the other with the inadvertent influence of the order in which one computes the throughput and aggregates the data statistically.

\section{FITTS' LAW}

Fitts' law (Fitts, 1954; Fitts \& Peterson, 1964) is a well known empirical regularity which predicts movement time $(M T)$ as a function of target width $(W)$ and target distance $(D)$. $\mathrm{HCl}$ researchers generally use the Shannon equation (MacKenzie, 1992; Soukoreff \& MacKenzie, 2004):

$$
M T=a+b \cdot \log _{2}(D / W+1)
$$

where $a$ and $b$ stand for adjustable constants and where the log term represents the task's index of difficulty $(I D)$.

In fact there are many candidate mathematical models for Fitts' law (see Plamondon \& Alimi, 1997, who list a dozen respectable equations), and not all models take the logarithmic form. In a famous contribution to the literature, Meyer et al. (1990) have proposed to model $M T$ as a power function of the ratio $D / W$, arguing that such a model encompasses the logarithmic model as a limiting case. This argument, however, has been recently challenged by Rioul and Guiard (2012), who showed that mathematically Meyer et al.'s model is a quasi-logarithmic, not a genuine power model. Not only is Equation 1 of the logarithmic category, not only is it known to tightly fit most data sets, it is also of special importance in practice, being 
actually part of an International ISO standard (ISO9241-9, 2002).

In this paper we re-examine the calculation of throughput (TP) from Equation 1 and draw attention to a previously unnoticed methodological difficulty that may hinder the empirical evaluation of the model.

\section{LAW OF VARIATION VS. INVARIANCE}

The Shannon model of Fitts' law is usually written as in Equation 1, which states a law of variation of the form $y=a+b x$. That is, $y$ varies lawfully (linearly) with $x$. But the model may just as well be formulated as an invariance, as either

$$
\frac{y-a}{x}=b
$$

or

$$
y-b x=a,
$$

emphasizing that two quantities, $a$ and $b$, are invariant across the variations of $x$.

It is noteworthy that, despite their mathematical equivalence, the law-of-variation formulation of Equation 1 and the invariance formulations of Equations 2-3 place the model in markedly different positions with regard to the risk of empirical falsification (Platt, 1964; Popper, 1959). The Shannon equation (Equation 1) is in fact quite unlikely to be disproved by empirical data: at worst, one will obtain a disappointing fit, wondering whether one should continue to trust the model with an $r^{2}$ below .9, .8, or lower. But take the claim that $(y-a) / x$ must be independent of $x$, which has the form of a null hypothesis $\left(\mathrm{H}_{0}\right)$ : if the data plead for the rejection of $\mathrm{H}_{0}$, then one faces an empirical falsification of Equation 3-and, by implication, of Equation 1. Consistent with classic Popperian epistemology (Platt, 1964; Popper, 1959), this more challenging way of empirically testing the theory is commonplace in stronger domains of science like physics (Meehl, 1967).

\section{THE THROUGHPUT}

The throughput (TP) of Fitts' law tasks is a standard of measurement widely used in the $\mathrm{HCl}$ community as a tool to quantify user performance with different input devices and different interaction techniques.

In classical Fitts' law experimentation participants are instructed to perform their movements as fast as possible given the $I D$, with a certain (ideally constant) level of accuracy. Although many factors such as mood, fatigue and alertness, may influence the $T P$, the Shannon model of Fitts' law says that the TP should be conserved within participant across variations of task difficulty (nominal $I D$ ) and movement accuracy (effective ID). Because the TP is a global index of performance which takes both speed and accuracy into account, its practical utility in the context of $\mathrm{HCl}$ research is very high.

MacKenzie and Isokoski (2008) recently tested the robustness of the TP under three different instructional conditions: standard, speed emphasis, and accuracy emphasis. While, unsurprisingly, speed and accuracy of performance were both strongly affected by the change of instructions, the key outcome was the authors' failure to detect a significant effect of the instructional manipulation on the TP. Mackenzie and Isokoski argued that this result is evidence for the Shannon model of Fitts' law.

Our purpose below is two-fold. First we reanalyze the data of a recently published study (Guiard et al., 2011) to test the null hypothesis of TP invariance across speed/accuracy variations. It occurred to us that because MacKenzie and Isokoski (2008) varied instructions within a limited range, their test of the Shannon model of Fitts' law was somewhat lenient. Obviously, the issue being the demonstration that a certain experimental manipulation exerts no effect on a certain dependent measure, the larger the extent of the manipulation, the more persuasive the demonstration. Thus, while our analysis below reproduces MacKenzie and Isokoski's lenient test on some fresh data, we will also report the results of a much tougher test in which the speed/accuracy strategy of our participants was made to vary over its whole spectrum, from a maximum-speed to a maximum-accuracy effort.

Our second purpose is to draw attention to a methodological difficulty that many authors may have incidentally noticed, without paying much attention to it, but that the results of present study forced us to consider seriously. The difficulty arises from the fact that the order in which one does the various operations required for the calculation of the TP affects the outcome to an appreciable extent. We will show that the problem is due to a mathematical result known as Jensen's inequality.

Twenty years have passed since MacKenzie (1992) first proposed to replace Fitts' original equation $M T=a+b \cdot \log _{2}(2 D / W)$ with Equation 1. MacKenzie has convinced the $\mathrm{HCl}$ community that the Shannon formula is theoretically valid and empirically predictive, but there is still no agreement on the exact definition of the TP.

While Zhai (2004) identified three candidate definitions in the literature, the basic dispute boils down to a simple mathematical dichotomy. What is not agreed upon is whether in the TP calculation 
one should take into account the intercept $a$ of the Shannon equation (Equation 1) and thus calculate the TP as

$$
T P_{\mathrm{Z}}=\frac{1}{b}=\frac{I D}{M T-a}
$$

or one should ignore the intercept and, in keeping with Fitts' (1954) initial suggestion, calculate the TP as

$$
T P_{\mathrm{M}}=\frac{I D}{M T}
$$

Equation 4 is a straightforward derivation of Equation 1. It gives the definition of the TP that Card et al. (1978) used in their well-known pioneering study of Fitts' law in the context of $\mathrm{HCl}$. More recently that definition was forcefully advocated by Zhai (2004), hence the subscript $Z$.

As emphasized by Zhai, Equation 5 is inconsistent with Equation 1, whose intercept $a$ it leaves aside. Nevertheless this definition of the TP has been inflexibly advocated by MacKenzie (hence the subscript $M$ ), based on the argument that in principle this intercept should be zero (Soukoreff \& MacKenzie, 2004). Recently Guiard and Olafsdottir (2011) argued that no sensible assumption regarding the value of Fitts' law intercept can be made because the $I D$ runs on a non-ratio scale of measurement (i.e., an equal-interval scale with no physical zero), meaning that the value of the intercept is arbitrary and uninterpretable. But the fact is, the $T P_{\mathrm{M}}$ has never ceased to be popular among $\mathrm{HCl}$ researchers and its credibility is now further strengthened by an ISO standard (ISO9241$9,2002)$.

In a recent study Wobbrock et al. (2011) warned against comparisons across the two categories of $T P$, which necessarily produce more or less discrepant estimates. But unfortunately there is room within one and the same approach for quite another sort of discrepancy. To make this point below we will stick to MacKenzie's definition of the TP (Equation 5). Consider Equations 6 and 7, two concrete statistical implementations of the mathematical formula of Equation 5:

$$
\begin{aligned}
& \overline{T P}_{\mathrm{M}, \mathrm{CtA}}=\frac{1}{N} \sum_{i=1}^{N} T P_{i} \\
& \overline{T P}_{\mathrm{M}, \mathrm{AtC}}=\frac{\overline{I D}}{\overline{M T}}=\frac{\log _{2}\left(1+\frac{\bar{A}}{4.133 \cdot \sigma_{A}}\right)}{\overline{M T}}
\end{aligned}
$$

The only difference lies in the order in which one performs the averaging and the computing: in Equation 6 one first computes a number of TP values and then averages them (the CtA order), while in Equation 7 one first averages the IDs and the MTs and then computes one value of TP (the AtC order). Both equations seem to be mathematically and statistically sound and researchers who have utilized both versions may have considered them equivalent. The TP description offered by the International ISO9241-9 standard hesitates between them. In our view there is reason to be concerned by this irresolution.

\section{FIRST AGGREGATE THEN COMPUTE OR THE REVERSE ORDER: A JENSEN'S INEQUA- LITY ISSUE}

To reiterate, the calculation of TP involves two sorts of operations. One is averaging, a statistical operation that compresses a set of numbers into a single summary value, typically a mean. The other is computing (e.g., calculating the quotient of a fraction), an arithmetic operation that also often combines several numbers into a single result. Unfortunately, the final TP value depends on the order in which the averaging and the computing are done, as shown in Figure 1 with a very simple numerical example.

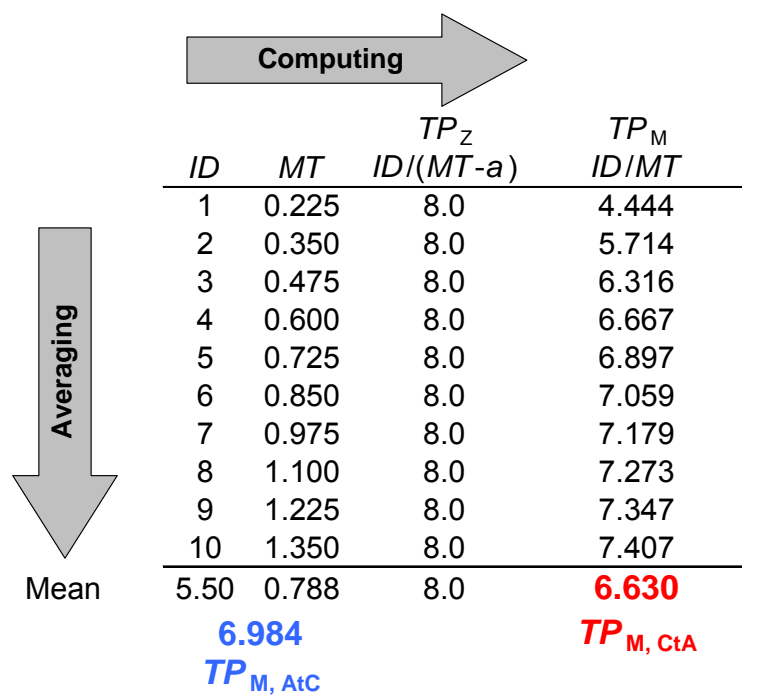

Figure 1. Two ways of calculating the $T P_{\mathrm{M}}$ under the assumption that Fitts' law follows the Shannon formula, with $a=0.1 \mathrm{~s}$ and $b=0.125 \mathrm{~s} / \mathrm{bit}$. The third column $T P_{Z}=$ $I D /(M T-a)$ is constant by hypothesis.

Figure 1 displays a hypothetical set of ten MT values computed from Equation 1 whose coefficients have been set to arbitrary but plausible values, $a=0.1 \mathrm{~s}$ and $b=0.125 \mathrm{~s} / \mathrm{bit}$ (Zhai, 2004). The figure helps to see that there are two ways to obtain a global value of $T P_{\mathrm{M}}$ from ten pairs of $I D$ and $M T$ values. One option is to start by computing $T P_{\mathrm{M}}$ in each row and to then average the ten 
values of $T P_{\mathrm{M}}$ at the bottom of the rightmost column-this is what we call the Compute-thenAverage (CtA) option. With the hypothetical data set of Figure 1 one obtains $T P_{\mathrm{M}}=6.63 \mathrm{bits} / \mathrm{s}$.

The alternative option is to start by averaging the $10 I D s$ and the $10 M T s$ downward and to then compute the $T P_{\mathrm{M}}$ just once from the mean $I D$ and the mean MT-this is what we call the Averagethen-Compute (AtC) option. With the data of Figure 1 this option yields $T P_{\mathrm{M}}=6.98 \mathrm{bits} / \mathrm{s}$, which is more $(+5.3 \%)$ than $6.63 \mathrm{bits} / \mathrm{s}$.

The problem one is encountering here is Jensen's inequality, which states that for any convex function

$$
f(\bar{x}) \leq \overline{f(x)}
$$

while the opposite holds true if the function is concave.

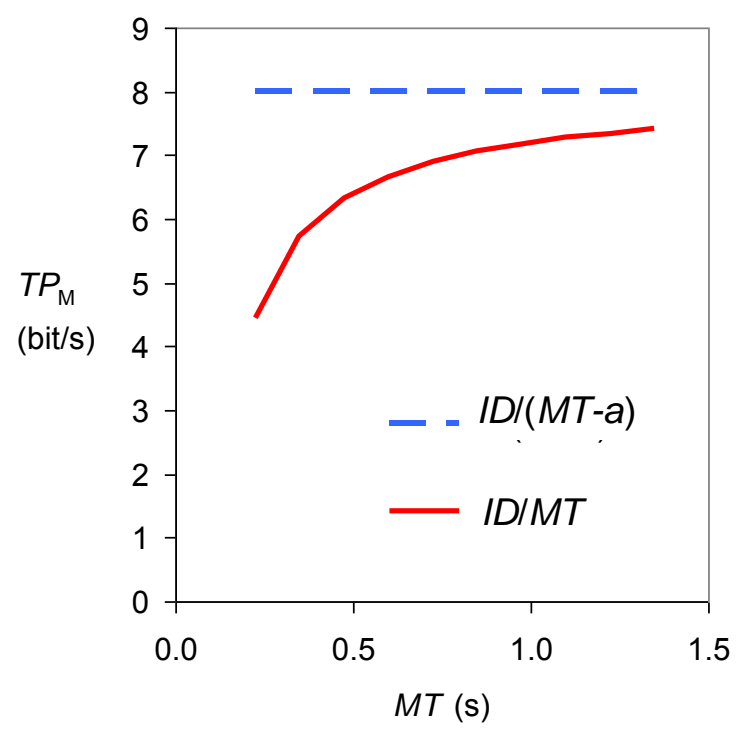

Figure 2. The function $T P_{\mathrm{M}}=f(M T)$ under the Shannon model.

Figure 2, which plots the data of Figure 1, shows that the function $T P_{\mathrm{M}} / T P_{\mathrm{Z}}=\mathrm{f}(M T)$, or $\mathrm{f}(M T)=1-$ $a / M T$, is concave, thus

$$
\overline{T P}_{\mathrm{M}, \mathrm{AtC}} \geq \overline{T P}_{\mathrm{M}, \mathrm{CtA}}
$$

Let us return to real-world formulas like those of Equations 6 and 7. The impact of Jensen's inequality is complex and rather hard to guess for two reasons.

First, the $T P_{\mathrm{M}}$ formula involves not one, but five calculation steps. Since computing the $T P_{\mathrm{M}}$ involves a function of the form

\footnotetext{
${ }^{1}$ A function is said to be convex (concave) if its graph lies above (resp. below) any tangent line.
}

$$
\frac{\log \left(1+\frac{x}{\sqrt{y}}\right)}{t}
$$

the computation of the $T P_{\mathrm{M}}$ requires five computation steps, represented from left to right in Figure 3.

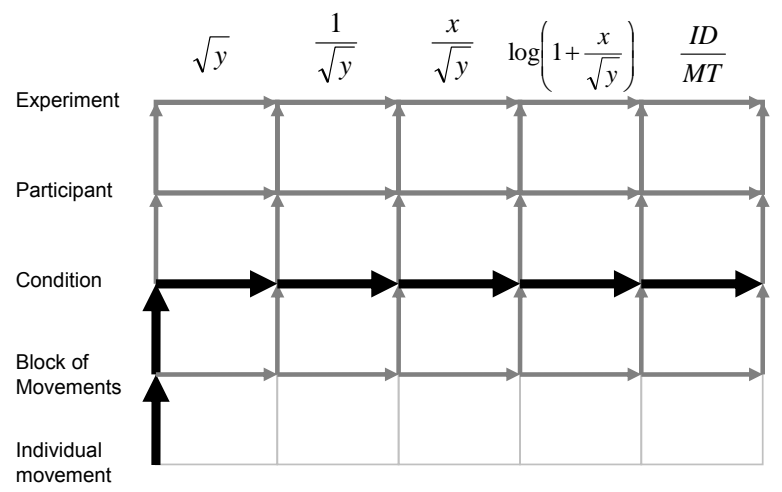

Figure 3. The statistical-aggregation and calculation steps involved in the computation of a $T P_{\mathrm{M}}$. One example path is highlighted, which delivers the $T P_{\mathrm{M}}$ value of one particular participant for one particular condition.

Second, as illustrated in Figure 3, a data set normally involves more than two levels of statistical aggregation. In fact the calculation of a $T P_{\mathrm{M}}$ may require up to four aggregation steps in a typical Fitts' law experiment (allowing for averaging over participants). Starting from the individual measure of MT-the atoms, so to speak-four successive aggregation steps, represented in the figure from bottom to top, can take place in a typical Fitts' law experiment:

a. the movement-block averages, each of which summarizes a number of individual measures;

b. the condition averages, each of which summarizes performance over a number of trial blocks;

c. the participant averages, each of which summarizes performance over a number of conditions; and

d. the experiment averages, each of which summarizes performance over a number of participants.

The important fact is that, contrary to the feeling that may arise from the simple comparison of Equations 6 and 7, there are many more than two ways of arranging the various computation and aggregation steps involved in the estimation of $T P_{\mathrm{M}}$. The number of paths we are looking for is the number of possible ways of inserting 3 objects in 6 possible places 


$$
\left(\begin{array}{l}
6 \\
3
\end{array}\right)=\frac{6 \times 5 \times 4}{3 \times 2 \times 1}=20
$$

Thus, with five computation steps and four aggregation levels, there exist 20 possible paths, which all deliver different $T P_{\mathrm{M}}$ values. ${ }^{2}$ Thus the Jensen inequality has many opportunities to operate, leading to a troublesome amount of uncontrolled variability in data processing.

The concrete example of the next section will show that this bias may be quite damaging. Depending on the CtA vs. AtC order, we found that our lenient test either succeeded or failed to replicate MacKenzie and Isokoski's (2008) result.

\section{A RERUN OF MACKENZIE AND ISOKOSKI'S TEST}

This section reports the results of a fresh test of the $T P$-invariance hypothesis based on a re-analysis of recently published data of ours (Guiard et al., 2011). Our experimental test differs from MacKenzie and Isokoski's (2008)—and by the same token from most standard Fitts' law tests-in three noteworthy respects.

First, we used discrete rather than reciprocal movements to obtain more reliable estimates of MT. As noticed by Fitts and Peterson (1964), the discrete protocol allows more rigorous control over the variables of interest than is possible with the reciprocal protocol. In the reciprocal protocol movement time is the time it takes to carry out a movement and to evaluate the error inherited from the previous movement and to prepare the next movement. The discrete protocol, in contrast, measures the duration of a pure movementexecution process.

Second, the target was displayed as a one-pixel line, rather than as a band of width $W$. This feature does not mean that the experiment used a zerowidth target, but rather that $W$ was left unspecified, the one-pixel target serving to just indicate to participants what the amplitude of their movements should be on average. Accordingly, in our calculations the $I D$ was computed from the ratio of mean movement amplitude (in fact always virtually equal to target distance $D$ ) to the standard deviation of the amplitude (rather than target width $W$. While the usual methodology uses $D$ and $W$ with a post-hoc adjustment for error because $W$ provides a notoriously poor control over the actual

\footnotetext{
${ }^{2}$ This calculation takes account of the fact that the very first operation can only consist of an aggregation, because the standard deviation of movement amplitude is undefined below the level of the block of movements.
}

spread of movement endpoints (e.g., Soukoreff \& MacKenzie, 2004), our strategy is to forget once and for all about any tolerance specification and to simply consider actual spreads of movement endpoints.

Third, and perhaps most importantly, our manipulation covered the complete range of speed/accuracy strategies, allowing a tougher and hence more informative empirical test of the Shannon model of Fitts' law.

\subsection{Method $^{3}$}

Sixteen participants were presented with five sets of instructions, which formed an ordinal independent variable:

- max speed

- $\quad$ speed emphasis

- speed/accuracy balance

- accuracy emphasis

- max accuracy.

In the max-speed condition the only accuracy requirement was to terminate the movements on average in the vicinity of the target. At the opposite end of the instructions continuum, in the maxaccuracy condition participants were to bring the cursor exactly to the target (zero pixel error), the only time constraint being to not waste any time. The three central levels of instructions, one unbiased (speed/accuracy balance) and two biased (speed emphasis and accuracy emphasis) were similar to those of MacKenzie and Isokoski.

The experiment used a computer screen and a Wacom ${ }^{\mathrm{TM}}$ tablet set to the absolute mode with a one-to-one mapping. The screen displayed two fixed vertical lines, $150 \mathrm{~mm}$ apart, indicating movement start and movement target, and a movable crosshair whose horizontal motion was controlled by the Wacom ${ }^{\mathrm{TM}}$ stylus. An L-shaped ruler was attached to the tablet to guide the stylus movement along the horizontal dimension, the shorter (vertical) leg of the $L$ being aligned with the screen's start line, thus eliminating start point variability.

Each of the 16 participants ran five 15-movement blocks in each of the five instructional condition (25 blocks overall). In sum this experiment involved 15 movements $\times 25$ blocks $\times 16$ participants $=6,000$ movements.

\subsection{Data Analysis}

We ran two within-participant one-way ANOVAs on $T P_{\mathrm{M}}$. In one of them, aimed to replicate the lenient

${ }^{3}$ For a detailed description of the method, see Guiard et al. (2011). 
test of MacKenzie and Isokoski, the instructions factor was restricted to its three central levels, namely speed emphasis, speed/accuracy balance, and accuracy emphasis. The other ANOVA considered all five levels, providing a much tougher test of the TP invariance hypothesis.

Individual-movement measures were $M T$ (s) and amplitude $(\mathrm{mm})$. For each or the 25 blocks we computed the three ingredients needed to calculate any $T P$, namely, median $M T$ and the mean and standard deviation of amplitude. ${ }^{4}$ We then computed the condition-level estimates of $T P_{\mathrm{M}}$ using both the CtA and the AtC order, ending up with two candidate dependent variables for the ANOVA test, $T P_{\mathrm{M}, \mathrm{CtA}}$ and $T P_{\mathrm{M}}$, Atc. The figures below show averages computed over all 16 participants.

\subsection{Results and Discussion}

Mean Amplitude

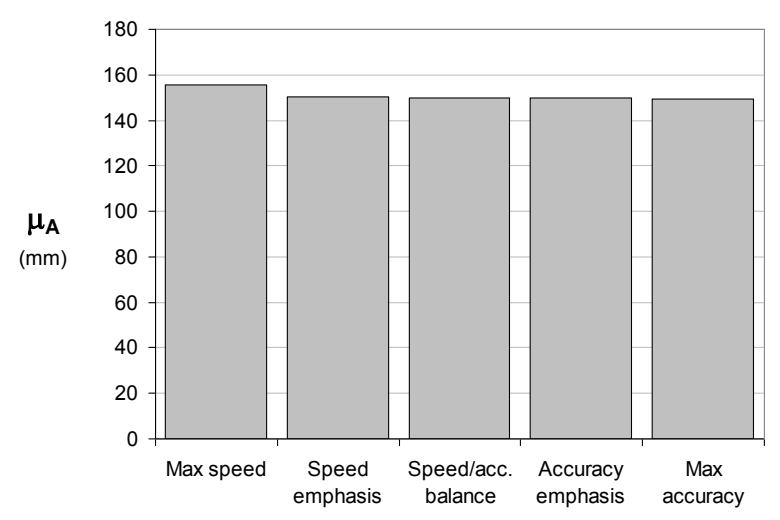

Figure 4. Mean amplitude vs. instructional condition.

As shown in Figure 4, mean movement amplitude $\left(\mu_{A}\right)$ was very nearly a constant $150 \mathrm{~mm}$, as required. The participants were able to produce essentially unbiased aiming movements, the only exception being a $5.5 \mathrm{~mm}$ overshoot error in the max-speed condition; although a statistically significant effect $\left(t_{15}=4.50, p<.001\right)$ this is a remarkably small bias of $+3.7 \%$, which we shall not discuss here.

Rather than movement amplitude, what our instructional manipulation did influence were, unsurprisingly, the speed and accuracy of performance, two very strong effects just as they were in the MacKenzie and Isokoski (2008) study. Figure 5 shows the gradual increase of median movement time $\left(\mu_{T}\right)$ from the max-speed condition (about 200ms) to the max-accuracy condition (more than a second), a considerable five-fold

4 The distributions of movement time showing some positive skewness, we used the median, rather than the mean, for that dependent measure. increase. Obviously a monotonic lengthening of movement time at a constant level of amplitude means a monotonic drop of average movement speed, shown in Figure 6.

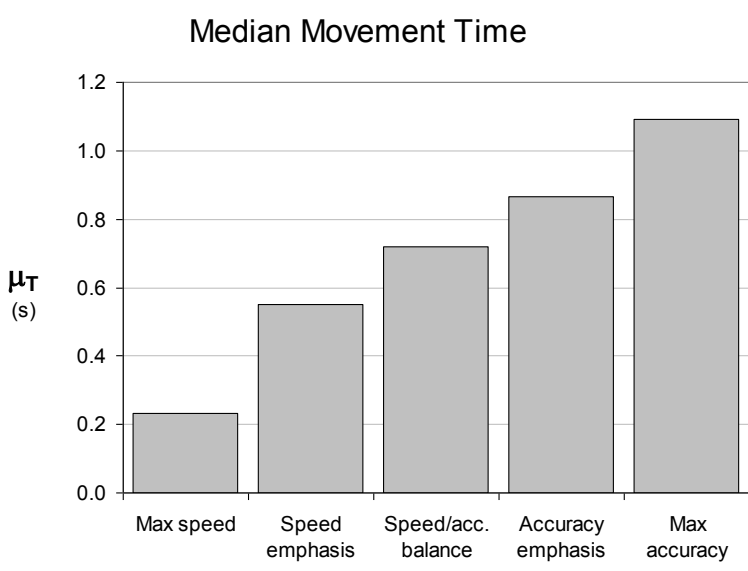

Figure 5. Median movement time as a function of instructional condition.

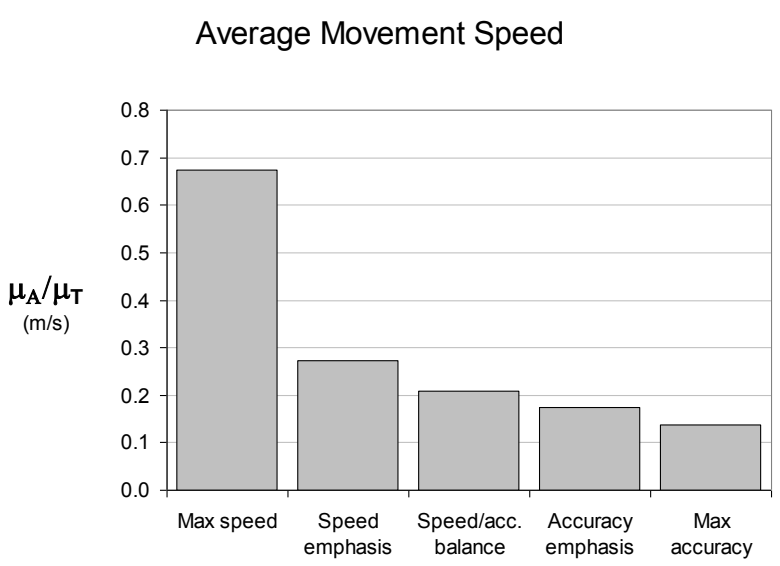

Figure 6. Average movement speed as a function of instructional condition.

Standard Deviation of Amplitude

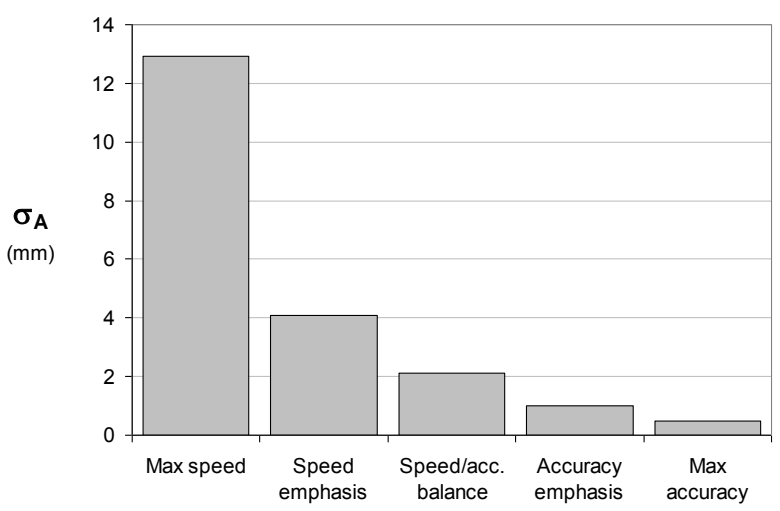

Figure 7. Endpoint spread as a function of instructional condition

The other side of the evidence that our instructions were instrumental in modulating the participants' strategy is visible in Figure 7 , which shows how 
the spread of movement endpoint, measured as the standard deviation of amplitude $\sigma_{\mathrm{A}}$, declined gradually from the max-speed condition (with a standard deviation of amplitude $\sigma_{\mathrm{A}}$ of $13 \mathrm{~mm}$, or $8 \%$ of the mean) to the max-accuracy condition $(0.5 \mathrm{~mm}$, or $0.3 \%$ of mean amplitude).

The crucial result of this experiment is shown in Figure 8, which plots $T P_{\mathrm{M}, \mathrm{CtA}}$ and $T P_{\mathrm{M}, \mathrm{AtC}}$, the two variants of the ISO estimate of $T P$, against the instructional factor.

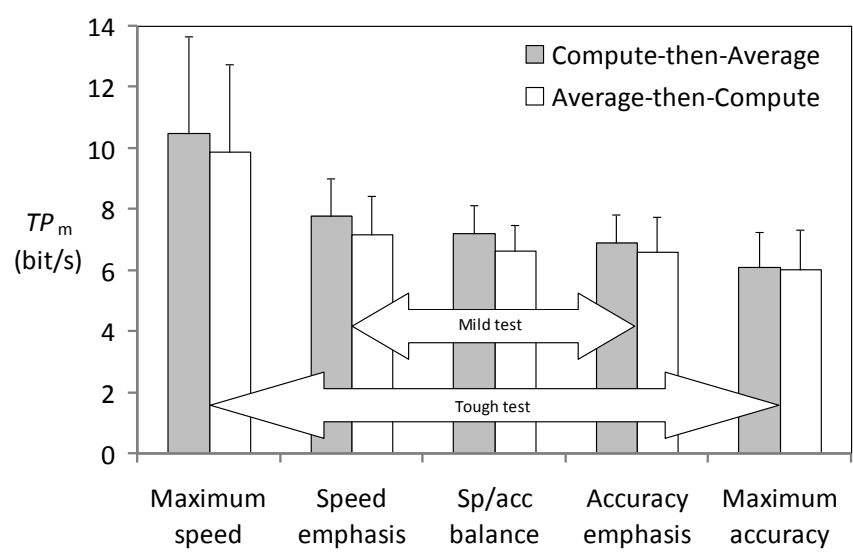

Figure 8. $T P_{\mathrm{M}}$ vs. instructional condition. Error bars show $95 \%$ confidence intervals based on betweenparticipant standard deviations.

Recall that according to MacKenzie and colleagues the $T P_{\mathrm{M}}$ should not vary across variations of the speed/accuracy strategy. Tested over our complete set of instructions from maximum speed to maximum accuracy, the $T P_{\mathrm{M}}$ invariance hypothesis markedly failed. Whether computed with the CtA or the AtC order, the $T P_{\mathrm{M}}$ declined monotonically, from about 10bits/s down to about 6bits/s, as the instructions were shifted from the max-speed to the max-accuracy condition. This is a substantial effect, a $42 \%$ reduction of the $T P$, and it is highly significant statistically (Table 1).

Table 1. Results of the tough and lenient ANOVAs conducted on the CtA and the AtC estimate of $T P_{\mathrm{M}}$.

\begin{tabular}{|c|c|c|c|c|c|c|}
\hline & \multicolumn{3}{|c|}{ Tough 5 level } & \multicolumn{3}{c|}{ Lenient 3 level } \\
\cline { 2 - 7 } & $F$ & $d f$ & $p$ & $F$ & $d f$ & $p$ \\
\hline CtA & 23.54 & 4 & $<.001$ & 5.03 & 2 & .013 \\
\hline AtC & 19.69 & 4 & $<.001$ & 1.86 & 2 & .173 \\
\hline
\end{tabular}

Turning to the lenient 3-level test, the outcome turned out to be equivocal. With the AtC order our lenient test replicated MacKenzie and Isokoski's non-rejection of $\mathrm{H}_{0}(p>.05)$. With the CtA order, however, it did not $(p<.02)$. This irresolution is a troublesome complication induced, we suggest, by Jensen's inequality.

In our view it is not necessarily the Shannon model of Equation 1 that should be questioned in light of the present results, but rather Equation 5, which requires the assumption that the intercept of Fitts' law is zero-actually an untenable assumption given the non-ratio level of measurement on the continuum of $\mu_{\mathrm{A}} / \sigma_{\mathrm{A}}$ or $D / W$ (Guiard \& Olafsdottir, 2011).

Would the test have been successful if the $T P_{\mathrm{Z}}$ had been used instead? We found with a simulation on our data set that the effect of instructions on TP would have been small and marginally significant, had the $T P_{\mathrm{Z}}$ been used instead of the $T P_{\mathrm{M}}$. This result is doubtful, however, because a test of the invariance of $T P_{Z}=1 / b$ across variations of the speed/accuracy strategy requires the other coefficient, the intercept $a$, to be used in the calculation. This requires that the Shannon equation be calculated beforehand, and so the test begs the question. Another sort of experimental test is needed to evaluate the invariance of the $T P_{Z}$.

\section{CONCLUSION AND PERSPECTIVES}

Routine $T P_{\mathrm{M}}$ measurement is an established norm of $\mathrm{HCl}$, further strengthened since 2002 by an official ISO standard. There is no question that standardization, which facilitates comparisons, is useful (Soukoreff \& MacKenzie, 2004). Twenty years of consensus about the Shannon model of Fitts' law have certainly been an asset for input research in $\mathrm{HCl}$. However, failure to acknowledge Zhai's (2004) demonstration that the standard method of measuring the TP (Equation 5) is inconsistent with the Shannon model (Equation 1) has been a handicap. Our data, which show that the $T P_{\mathrm{M}}$ not simply fails a tough invariance test but hardly passes a rather lenient test, support Zhai's (2004) suggestion that researchers should return to the mathematically correct definition of the TP shown in Equation 4.

Another, no less important lesson to be learned from this study is that serious methodological work is needed to try to master the hidden variability that arises inadvertently in Fitts' law data due to Jensen's inequality. To our knowledge the impact of this methodological difficulty on data processing has not been yet correctly understood and we believe this general problem is worth a systematic investigation. There is reason to believe that this is a general methodological problem whose scope presumably extends far beyond the study of Fitts' law. Whether the solution rests on some mathematical or statistical principles or perhaps on some arbitrary conventions is an open question which we are currently investigating. 


\section{REFERENCES}

Card, SK, English, WK, \& Burr, BJ, (1978) Evaluation of mouse, rate-controlled joystick, step keys and text keys for text Selection on a CRT. Ergonomics, 21, 601613.

Fitts, PM (1954) The information capacity of the human motor system in controlling the amplitude of movement. Journal of Experimental Psychology, 47, 381-391. Reprint: Journal of Experimental Psychology: General, 1992, 121, 262-269.

Fitts, PM, \& Peterson, JR (1964) Information Capacity of Discrete Motor Responses. Journal of Experimental Psychology, 67,103-112.

Guiard Y., \& Olafsdottir, HB (2011). On the measurement of movement difficulty in the standard approach to Fitts' law. PLoS ONE 6(10): e24389.

Guiard, Y, Olafsdottir, HB, \& Perrault, ST (2011) Fitts' law as an explicit time/error trade-off. Proc. CHI 2011. New York: ACM, 1609-1628.

ISO (2002). Reference Number: ISO 9241-9: 2000(E): Ergonomic requirements for office work with visual display terminals (VDTs) - Part 9 - Requirements for non-keyboard input devices (ISO 9241-9) (Vol. February 15, 2002): International Organisation for Standardisation.

MacKenzie, IS (1992) Fitts' law as a research and design tool in human-computer interaction. Human-Computer Interaction, 7, 91-139

MacKenzie, IS, \& Isokoski, P (2008) Fitts' throughput and the speed-accuracy tradeoff. Proceedings of CHI'2008. New York: Sheridan, 1633-1636.

MacKenzie, IS, Jusoh, S (2001) An evaluation of two input devices for remote pointing. Proceedings of the Eighth IFIP Working Conference on Engineering for Human-Computer Interaction$\mathrm{EHCl}$ 2001. Springer, Heidelberg, Germany, 235-249.

MacKenzie, IS, Soukoreff, RW (2003) Card, English, \& Burr (1978)-25 years later. Extended Abstracts of the ACM Conference on Human Factors in Computing Systems, CHI 2003, 760761.
Meehl, PE (1967) Theory testing in psychology and physics: A methodological paradox. Philosophy of Science, 34, 103-115.

Meyer, DE, Smith, JEK, Kornblum, S, Abrams, RA, \& Wright, CE (1990) Speed-accuracy trade-offs in aimed movements: Toward a theory of rapid voluntary action. In M. Jeannerod (Ed.), Attention and performance XIII (pp. 173-226). Hillsdale, NJ: Erlbaum.

Plamondon, R, \& Alimi, AM (1997) Speed/accuracy trade-offs in target-directed movements. The Behavioral and Brain Sciences, 20, 279-349.

Platt, JR (1964) Strong inference. Science, 146, N 3642, 347-353.

Popper, KR (1959) The Logic of Scientific Discovery. New York: Basic Books.

Rioul, O \& Guiard, Y (2011) "The power model of Fitts' law does not encompass the logarithmic model." 2011 Meeting of the European Mathematical Psychology Group (EMPG 2011) Paris, France, August 29-31, 2011.

Rioul, O \& Guiard, Y (in press) Power vs. logarithmic model of Fitts' law: A mathematical analysis. Mathematics and Social Sciences.

Soukoreff, RW, \& MacKenzie, IS (2004) Towards a standard for pointing device evaluation: Perspectives on 27 years of Fitts' law research in $\mathrm{HCl}$. International J. Human Computer Studies, 61, 751-789.

Wobbrock, JO, Shinohara, K, \& Jansen, A (2011) The effects of task dimensionality, endpoint deviation, throughput calculation, and experiment design on pointing measures and models. Proc. CHI 2011. New York: ACM, 1639-1648.

Zhai, S (2004) Characterizing computer input with Fitts' law parameters: The information and the non-information aspects of pointing. International Journal of Human Computer Studies, 61, 791-809. 